\title{
Biomagnetic methodologies for the noninvasive investigations of the human brain (Magnobrain)
}

\author{
George Anogianakis*a,d, G.F.A. Harding ${ }^{\mathrm{b}}$, Maria Peters ${ }^{\mathrm{c}}$, Michael Apostolakis ${ }^{\mathrm{i}}$, \\ George Foroglou ${ }^{e}$, Juergen Vieth ${ }^{r}$, Andy Ioannides ${ }^{g}$ \\ "Department of Physiology. \\ "Neurosurgery Clinic, Aristotle University of Thessaloniki. Thessaloniki, 54006 Greece \\ ${ }^{h}$ Department of Vision Science, Aston Unitersit1, Birmingham. UK \\ 'Department of Technical Physics, University of Twente, Enschede, The Netherlands \\ "BIOTRAST UETP, 111 Mitropoleos Str., Thessaloniki, 54622 Greece \\ Department of Neuropsychiatry, University of Erlangen, Erlangen, Denmark \\ "Department of Physics, Open University. Milton Keynes. UK
}

\begin{abstract}
Magnetoencephalography (MEG) non-invasively infers the distribution of electric currents in the brain by measuring the magnetic fields they induce. Its superb spatial and temporal resolution provides a solid basis for the 'functional imaging' of the brain provided it is integrated with other brain imaging techniques. MAGNOBRAIN is an applied research project that developed tools to integrate MEG with MRI and EEG. These include: (1) software for MEG oriented MRI feature extraction; (2) the Brain Data Base (BDB) which is a reference library of information on the brain used for more realistic and biologically meaningful functional localisations through MEG and EEG; and (3) a database of normative data (age and sex matched) for the interpretation of MEG. It is expected that these tools will evolve into a medical informatics environment that will aid the planning of neurosurgical operations as well as contribute to the exploration of mental function including the study of perception and cognition.
\end{abstract}

Key words: Magnetoencephalography; MRI; EEG; Epilepsy; Multiple sclerosis

\section{Introduction}

MEG is a non-invasive technique well suited for monitoring the electrical activity of the brain. It infers information about the electric currents of the brain by sensing the magnetic fields they induce through SQUIDs (Superconducting QUantun In-

\footnotetext{
* Corresponding author
}

terference Devices). Present instruments, despite their high cost, permit measurement of biomagnetic events on a millisecond time scale while they can localise their sources (neuronal populations) within a few millimetres. Therefore, MEG can become a powerful tool for 'functional imaging' of the brain provided it is integrated with other evaluation and imaging techniques for the brain, especially MRI [6]. MAGNOBRAIN was designed to provide informatics tools to achieve this integration. 
The explicit attempt by MAGNOBRAIN to integrate MEG with MRI and EEG implied the realisation of three operational goals:

(1) to provide tested software for MEG oriented MRI image processing and MRI feature extraction;

(2) to establish the BDB; and

(3) to create a database of normative data (age and sex matched) focused on epilepsy, acute optic neuritis/multiple sclerosis (MS) and optic neuropathy.

\section{Methods}

All MAGNOBRAIN software was developed to run on a high-end PCs, under Windows 3.1. Computer graphics were resorted to for directly visualising neuroanatomical information. This is of paramount significance since 3D neuroanatomical relations, along with information about the brain microstructure, are important in interpreting neurophysiological data.

The BDB is an example of a relational database for data on local brain microstructure. Since relevant data are extremely scarce, MAGNOBRAIN spend major efforts to produce the necessary comprehensive data set through the systematic sectioning of three fresh human brains (aged 20-40 years) from the cadavers of car accident victims who were brought to the Thessaloniki morgue. The procedure that was followed included the preparation of cortical material obtained from these brains (and covering every Brodmann field of the cortex) for light microscopy. Microscope slides thus prepared were then photographed through a video camera mounted on a microscope. The images were subsequently analysed by a dedicated digital image processor. The information that was extracted concerned the relative differences in pyramidal cell densities across the human cortex, the pyramidal cell size and pyramidal cell surface area distribution over the visual cortex as well as data on the form of the pyramidal cells.

The final operational goal of MAGNOBRAIN was the creation of a database of normative data focused on epilepsy, acute optic neuritis/multiple sclerosis and optic neuropathy. Epilepsy is divided into focal, generalised and epilepsy with secondary generalisation. In contrast to the wealth of EEG data on all three types of epilepsy, only a handful of cases of focal epilepsy have been studied with MEG. To redress this situation, MAGNOBRAIN collected data on photosensitive epilepsy, mainly, but also on other focal types of epilepsy. Photosensitive epilepsy was targeted because drugs that block the secondary discharge leave the primary focal activity largely unaffected and permit the in-depth investigation of the primary focal anomaly.

Visual Evoked Potentials (VEPs) are used in the investigation of MS. Relatively few studies have been carried out on Acute Optic Neuritis, however. Since the demyelination affects either P-type or Mx-type fibers, MEG (especially event related changes in MEG), can provide an inside to the precise cortical contribution of the normal response. Within MAGNOBRAIN combined EEG, MEG and VEP studies of selected patients were carried out and correlations with MRI findings were sought. Similar investigations were carried out on optic neuropathy. Finally, data were collected from age and sex matched normals.

\section{Results}

\subsection{Software for MEG oriented MRI image pro- cessing and MRI feature extraction}

The MAGNOBRAIN efforts in specialised MRI image processing and feature extraction have resulted in the production of two sets of software tools: (1) the BRAINQUERY environment; and (2) the MAGNOBRAIN MRI segmentation tool. Although each of these tools represents an independent entity within MAGNOBRAIN they served as primary sources of information for building the BDB.

BRAINQUERY [3] is an environment that intelligently processes MRI data and 'extracts' anatomical and physiological information about brain structures by querying suitably chosen experts (e.g., neurologists). The expert 'dissects' the brain (as it is reconstructed from the MRI sections) on the computer screen while he answers questions posed to him by the programme. The questions the expert is called to answer stem from the BDB entity-relationship diagrams. The MRI 
images used by BRAINQUERY are composed of $256 \mathrm{I}$-mm thick slices each containing $256 \times 256$ $\mathrm{mm}^{3}$ voxels. BRAINQUERY can handle a large number of windows, being limited only by memory. Each window can be an MRI slice in numerous formats (i.e., .TIF, .PCX, .GIF, etc.), or any other bitmap. Each window can be moved, resized to an arbitrary size or to three standard sizes (normal, double or device specific 'largest window' available). Finally, BRAINQUERY can print (or save) any of these images in numerous bitmap formats (e.g., .PCX, .GIF, .BMP) and exchange them with other programs for further processing. Present BRAINQUERY development centers on algorithms to permit the 'convolution' of any set of MRI slices onto the BDB anatomical information, automating thus MRI interpretation. The MAGNOBRAIN MRI segmentation tool can:

(1) display any arbitrary MRI slice from its collection of MRI slices;

(2) save the image into a file;

(3) compute the histogram of pixel intensity values;

(4) clear pixels whose intensity is greater than a chosen color limit;

(5) zoom into an area of the image;

(6) delete an area of the image;

(7) swap images among the active windows;

(8) add/subtract the image in one active window from that displayed in another;

(9) sharpen the image in the active window;

(10) smooth the image in the active window using four different types of spatial filtering;

(11) calculate the Laplacian or the Gradient of the image in the active window;

(12) exit from the application.

\subsection{The $B D B$}

$\mathrm{BDB}$ is an innovative database that keeps (in a structural way) those anatomical and functional features of the human brain that are suitable for increasing the localisation ability of biomagnetic techniques, especially in the case of epileptic foci or evoked brain activity $[2,4]$. The BDB contains electrophysiological, neuroanatomical, neuropathological, neurosurgical and radiological (mainly MRI) information on essential structures of the human brain. It handles complex and nonuniform 3D objects and admits efficient query evaluation through state-of-the-art computational geometry techniques. Thus BDB includes a complete anatomical description of the brain in $x, y$ and $z$ coordinates. To better organise this information, the human brain is divided into approx. $1.5 \times 10^{6} 1-\mathrm{mm}^{3}$ cubes whose features are described as attributes. The coordinates for each cube are based on a 3D proportional grid reference system which adapts to individual brains The attributes for each cube include:

(1) anatomical name of the brain area to which it belongs;

(2) origin(s) of inputs it receives;

(3) destination(s) of outputs it gives out:

(4) neuro-physio/psycho-logical functions that it subserves;

(5) global brain circuits that it belongs to;

(6) types of injuries or pathologies likely to occur there, including information on the accompanying symptoms, clinical signs and their functional assessment;

(7) microanatomical (quantified if possible) characteristics, e.g., types and density of neuronal and glial cells and types of neural fibers contained [5].

The efficient functioning of the BDB is based on the fact that necessary queries are geometrical in nature, involving the concepts of proximity and of topology. Indeed, the BDB contains 3D objects with various non-uniform shapes, which, in most cases, can be hierarchically linked to each other. Efficient data representations and quick access methods for these objects were developed through the adoption of the 'flexible box' concept. A flexible box is an orthogonal parallelepiped which includes a great number of basic cubes, the number of which is defined according to the needs of every case. Generally, the set of flexible boxes that parsimoniously represents a given brain volume is defined as the smallest possible number of non-overlapped orthogonal parallelepipeds (parallel to axes) of varying dimensions that contains that volume. Using the flexible box concept, a brain structure can be described very efficiently since it is not necessary to describe every voxel it contains but only a relatively small number of flexible boxes. 
BDB modelling of the different connections between different areas of the nervous system is based on the fact that fibers may be grouped into bundles which (1) branch off and (2) come in complex forms. Fibers of the same type which link the same brain areas, are defined as a 'fiber bundle'. Fiber bundles are composite objects, since they come in various non-uniform shapes, have variable thickness and are very long. In order to reduce the fibre bundle representation complexity, they are divided into fundamental segments, called 'bundle sections' which have no topological anomalies and, therefore, do not require special techniques for their modelling. Thus, in order to correlate topology with brain geography (i.e., to correlate bundle sections with the brain space they occupy), flexible boxes are used. The length of different bundles, which is of critical importance for interpreting neurophysiological data, can be efficiently estimated by using the concept of 'central path', i.e., that set of elementary BDB cubes each of which is central to a cross-section of a fiber bundle.

Since BDB entities are (in general) hierarchically connected, a tree structure diagram was adopted for representing the various $\mathrm{BDB}$ relationships while, due to the vast size of the information contained in BDB, space and time efficient (customised) solutions were developed to implement the necessary queries.

The BDB information has been compiled and organised in this way for the first time, and is not available anywhere else, at least on this scale. It includes the relative differences in pyramidal cell densities across the human cortex, the pyramidal cell size and the pyramidal cell surface area distribution over the visual cortex. At present, the BDB contains over 1000 microscopic scannings of the visual cortex or an aggregate of 2 Gbytes of data [5].

\subsection{The database on normative $M E G$ data}

The database of normative data for normal and abnormal subjects properly matched as to age and sex throughout the lifespan represents the last of the MAGNOBRAIN deliverables. It contains collections of EEG and MEG data from both normals and sufferers of Photosensitive Epilepsy, Optic Neuritis, MS and Optic Neuropathy collected according to strict protocols. In this way it is possible to both compare normal with abnormal responses and to compare the performance of different localisation algorithms on the same set of input data. Thus it is hoped that it will serve as the testbed for future development and standardisation in neuromagnetism.

\section{Discussion}

All along MAGNOBRAIN was perceived as a project in the spirit of the 'decade of the brain' (1991-2000) in that it was built on the premise that mapping the brain and its functions would become a main gateway to future brain research [1]. MAGNOBRAIN anticipated the fact that in the $90 \mathrm{~s}$ neural circuit databases would be composed of multiple components, including references and tools for matching or warping one brain image into another, and would be registries of available data. In fact, the BDB and the database of normative data that have been implemented within MAGNOBRAIN can be considered as prototypes of the neuronal circuit databases of the future.

\section{References}

[1] Report of the US Committee on a National Neural Circuitry Database Mapping the Brain and its functions/ Integrating enabling technologies into Neuroscience Research published by National Academy Press, Washington DC, 1991.

[2] G. Anogianakis, A. Tsakalidis, P. Spyrakis, K. Krotopoulou and D. Terpou, Satellite Symposium of Neuroscience Technology. Mapping the Brain in a Structural way helps in the localization of and Epileptic Foci. Lyon, France, November 2-4, 1992

[3] G. Anogianakis and G. Nendidis, An image processing environment for MRI that captures expert knowledge about the brain, 2nd European Conference on Engineering and Medicine, Stuttgart, April 1993.

[4] G. Anogianakis, K. Krotopoulou, D. Terpou, A. Tsakalidis and P. Spyrakis, Brain Data Base in, Database and Expert System Applications, Lecture Notes in Computer Science 720 , eds. Vladimir Marik, Jiri Lazansky and Roland Wagner, Springer-Verlag, 1993.

[5] G. Anogianakis, M. Kazamzadeh, A. Manthos, A. Verlygen, G. Nendidis, Ch. Foroglou, M. Apostolakis and G. Foroglou, Prototype of a computerized light microscopy atlas of the occipital cortex. Schweizerische Gesellschaft fur Anatomie, Histologie und Anatomie Proceedings, Fribourg 1993.

[6] G.F.A. Harding, How surgeons could navigate the brain, New Scientist - 11 December 1993. 